This item was submitted to Loughborough's Research Repository by the author.

Items in Figshare are protected by copyright, with all rights reserved, unless otherwise indicated.

\title{
Stagnation temperature measurement using thin-film platinum resistance sensors
}

PLEASE CITE THE PUBLISHED VERSION

http://dx.doi.org/10.1088/0957-0233/25/1/015101

PUBLISHER

(C) IOP Publishing

VERSION

AM (Accepted Manuscript)

\section{PUBLISHER STATEMENT}

This work is made available according to the conditions of the Creative Commons Attribution-NonCommercialNoDerivatives 4.0 International (CC BY-NC-ND 4.0) licence. Full details of this licence are available at: https://creativecommons.org/licenses/by-nc-nd/4.0/

\section{LICENCE}

CC BY-NC-ND 4.0

\section{REPOSITORY RECORD}

Bonham, Clare, Steven J. Thorpe, Mark N. Erlund, and Richard D. Stevenson. 2013. "Stagnation Temperature Measurement Using Thin-film Platinum Resistance Sensors". Loughborough University. https://hdl.handle.net/2134/17462. 


\title{
Stagnation temperature measurement using
}

\section{thin-film platinum resistance sensors}

\author{
C Bonham ${ }^{1}, \mathrm{~S}$ J Thorpe ${ }^{1}, \mathrm{M}$ N Erlund ${ }^{2}$ and R D Stevenson ${ }^{2}$ \\ ${ }^{1}$ Department of Aeronautical and Automotive Engineering, Loughborough \\ University, Loughborough, LE11 3TU, UK \\ 2 Rolls-Royce plc, PO Box 31, Derby, DE24 8BJ, UK \\ E-mail: c.bonham@lboro.ac.uk
}

\begin{abstract}
The measurement of stagnation temperature in high speed flows is an important aspect of gas turbine engine testing. The ongoing requirement to improve the accuracy of such measurements has led to the development of probe systems that use a thin-film platinum resistance thermometer (PRT) as the sensing element. For certain aspects of engine testing this type of sensing device potentially offers superior measurement performance to the thermocouple, the temperature sensor of choice in most gas turbine applications. This article considers the measurement performance of prototype PRT-based stagnation temperature probes, up to high subsonic flow conditions, using passively aspirated probe heads. The relatively poor temperature recovery performance of a simply constructed probe has led to the development of a new design that is intended to reduce the impact of thermal conduction within the probe assembly. The performance of this so-called dual-skin probe has been measured through a series of tests at a range of Mach numbers, incidence angles and Reynolds numbers. The data reveal that a high probe recovery factor has been achieved with this device, and that the application of this design to engine tests would yield the measurement performance benefits of the PRT whilst requiring small levels of temperature recovery compensation.
\end{abstract}


Keywords: stagnation temperature, resistance thermometry, recovery factor, PRT 


\section{Introduction}

During the development phase of a gas turbine engine significant attention is focused on the quantification and validation of the engine performance. This process primarily establishes whether any improvement/modification is required to meet the intended specification, and can also be used to improve the design methodology. As part of this process an extensive whole-engine test campaign is conducted during which an instrumented working prototype is used to obtain critical flow condition measurements that allow an assessment of the machine aero-thermal performance. Amongst the wide range of measurements that are usually obtained, the gas-path condition is primarily established through measurements of the stagnation temperature and pressure at various locations. These data are then used to determine parameters, such as the turbomachinery efficiency, which can be used to establish whether particular components are operating as intended. To continue the development of engines with improved efficiency, manufacturers need the capability to measure smaller increments of performance and this has a direct impact on measurement accuracy requirements. The flows within gas turbine engines are generally in the high-subsonic and transonic regimes, which leads to specific instrumentation requirements such that accurate stagnation quantities can be measured. Additionally, the probes must be sufficiently compact, robust and reliable for use in the harsh engine environment where factors such as vibration and foreign object damage are important. The current article considers a means for improving the accuracy of stagnation temperature measurements during whole-engine test campaigns, and in particular the application of platinum resistance thermometry.

Stagnation temperature instrumentation with specific application to high velocity flows has been developed over the last 80 years or so. It is well established that it is 
be passed over a temperature sensor in a controlled manner. In machine applications the stagnation temperature is generally measured using probes that adiabatically decelerate the high-speed flow to low velocity (Mach number typically less than 0.15 ), before this fluid is allowed to pass over a temperature sensitive element such as a thermocouple. The accuracy of the indicated stagnation temperature depends on a number of factors that includes the fundamental performance of the temperature sensitive element, the static calibration accuracy, temperature recovery effects of the flow over the sensor, and installation effects such as thermal conduction and radiation. A brief overview of relevant previous work in this field is provided below.

The work of Franz (1940) considered the application of thermocouples to the measurement of stagnation temperature in high-velocity supercharger flows; both aspirated and non-aspirated probe designs were tested, the former being referred to as a "through-flow device". In this early study it was recognised that thermal conduction is an important controlling parameter in the measurement performance of probe systems. Hottel and Kalitinsky (1945) considered the use of bare-wire thermocouples in high-speed flows, specifically investigating three shrouded designs (self-aspirated) that decelerate the flow to low velocity within the probe head. The performance of these probes was compared over a range of flow velocities and incidence angles. The primary data reported was the probe temperature recovery factor (defined in Section 3.2); values of between 0.9 and 0.98 were observed depending on the oncoming flow condition. Importantly, Hottel and Kalitinsky recognised that the "ventilation of the thermocouple junction needed to be sufficient to compensate for heat losses due to conduction and radiation". Glawe et al (1956) reported the investigation of nine bare-wire thermocouple probes of both passive and forced aspiration types. Willbanks (1973) reported the probe temperature recovery factor of a passively-aspirated thermocouple probe specifically 
and operating pressure (Mach and Reynolds numbers). One of the most comprehensive assessments of stagnation temperature instrumentation is that due to Moffat (1961) who presented an overall analysis of the thermal behaviour of aspirated thermocouple probes.

In an attempt to improve temperature measurement accuracy, Zeisberger (2007) has more recently presented an analysis of the performance of a thermocouple-based temperature rake for combustor and turbine applications in a gas turbine engine. This work considered the impact of various detrimental effects such as radiation, conduction and flow Mach number within a Kiel-type stagnation tube. A measurement accuracy of $0.4 \%$ of absolute stagnation temperature is claimed for Zeisberger's optimised design in typical turbine-like (high-temperature) applications.

The current article considers the development of a new PRT-based stagnation temperature probe for gas turbine engine use and, in particular, for regions where the flow stagnation temperature is close to ambient. Improvements due to this device have been designed to: exploit recent advances in the development of miniature thin-film PRT sensors, reduce the impact of thermal conduction within the probe assembly, and yield more accurate measurements in high subsonic Mach number flows. The device is described and test results are presented and discussed for various configurations. The reported data includes the effects of incidence angle, Mach number and Reynolds number. The performance of this new device is compared to a conventional probe constructed in similar fashion to typical current engine instrumentation hardware. Note that although the PRT sensors used in this study have a temperature capability up to $400^{\circ} \mathrm{C}$, the current probes are intended to be applied to the low pressure region of a gas turbine compression system, and in particular the fan of a large civil engine. In this region the gas stagnation temperature is close to ambient at stage inlet and a few tens 
Throughout this article reference is made to the indicated probe temperature, denoted by $T_{i}$. This is the temperature obtained from the probe after the application of any static calibration data, and is therefore the actual temperature of the sensing element. In general, this temperature is below the true stagnation temperature of the fluid in which the probe is immersed. In principle, this indicated temperature could be corrected such that a more accurate value of the gas stagnation temperature is established, although this procedure would require the application of aerodynamic calibration data, or the use of validated aero-thermal modelling analyses.

\section{Stagnation temperature measurements in gas turbine engines}

An important use of gas-path measurements is the determination of turbomachinery efficiency. Since the efficiency of particular engine components (such as the fan in a turbofan engine) has a direct impact on engine fuel consumption, feedback of the measured performance data is critical to the refinement and improvement of engine design methods. For example, the isentropic efficiency of a compression system can be deduced from measurements of the stagnation temperature rise and pressure ratio through equation 1 ,

$$
\eta=\frac{T_{o, \text { inlet }}}{T_{o, \text { inlet }}-T_{o, \text { exit }}}\left[P R^{\frac{\gamma-1}{\gamma}}-1\right]
$$

from which it is clear that the measurement of an accurate isentropic efficiency is sensitive to measurement uncertainties in both the inlet stagnation temperature and stage temperature rise. The sensitivity of the deduced efficiency to temperature measurement uncertainty can be appreciated from Fig. 1, which shows the impact of a $1 \mathrm{~K}$ error in stage temperature rise as a function of the compressor pressure ratio (based on equation 1). A single stage fan of a turbofan engine typically has a pressure ratio in the range 1.4 to 1.8 , and consequently an uncertainty in the measured 
Considerations such as these have lead to a desire within the gas turbine industry to improve the accuracy of measurements obtained during costly engine tests: uncertainties of $\pm 0.1 \%$ in absolute stagnation temperature are now being sought, compared to the current situation that is typically no better than $\pm 0.5 \%$. For fan inlet flow conditions this means overall measurement uncertainty from all sources of approximately $\pm 0.3 \mathrm{~K}$. Although the development of stagnation temperature instrumentation is relatively mature and adequate for most situations, it is this requirement to enhance engine test measurement accuracy that is driving new instrumentation development for high subsonic flow conditions.

As suggested by Moffat (1961) two distinct strategies can be used in order to improve the accuracy with which stagnation temperature measurements are made. Firstly, attempts can be made to design a probe assembly such that sufficiently accurate measurements are made without the need to conduct an aerodynamic calibration of individual probes. Secondly, the probe behaviour can be characterised in sufficient detail experimentally such that corrections can be made to the indicated temperature data to yield overall reduced error. The difficulties of the second approach led Moffat to conclude that it is best to adopt the first; in reality, however, a combination of both good design and aerodynamic calibration may be employed.

\subsection{Temperature sensing elements}

The measurement of stagnation temperature in gas turbine engines relies extensively on the use of various types of thermocouple. Typically these are either bare-wire (exposed junction) or encased in a type of sheath (e.g. stainless steel, nickel alloy) depending on the application. While thermocouples are well characterised and have high-temperature capability, they have some characteristics that are not desirable: 1) 
generated, 3) the affect of strain on the thermocouple wires, 4) the adverse impact of the inclusion of connectors and extension wire. Although in general it is possible to refine the performance of a thermocouple system through the use of high quality measurement equipment (such as the reference junction) and careful calibration of the measurement chain, the author's experience of using thermocouples on an engine testbed have shown that any potential improvement in accuracy is in practice extremely difficult to retain. In comparison, initial applications of the thin-film PRT in engine test campaigns have demonstrated high repeatability, good calibration retention and relative simplicity in application. This is considered to be related to the relatively high signal level of the PRT (at an appropriate excitation current the voltage-temperature level is typically an order of magnitude greater than that of a thermocouple), and the elimination of complexities surrounding the provision of an accurate reference junction in the engine test environment.

Although not currently in common use in gas turbine engine tests, the PRT is known to offer potential benefits in certain measurement applications. Traditionally, PRT devices have been used as secondary temperature standards in thermal calibration systems, these being constructed from platinum wire that is wound around a ceramic core. Platinum displays a stable, repeatable resistance-temperature response, making it ideal for superior accuracy measurement applications. However, the relatively large size of these sensors, and problems related to vibration in machine environments (such as gas turbine engines), have made the application of the wire-wound PRT problematic. The more recent availability of commercially produced miniature thin-film PRT devices has allowed the consideration of resistance thermometry in a wider range of applications. Thin-film PRT sensors consist of a ceramic substrate (typically alumina) onto which is deposited a thin platinum track. In common with other resistance thermometers, the 
$100 \mathrm{Ohms}$ ). The platinised side of the ceramic is then coated with a layer of glass that forms a protective barrier, and also covers the interface between the platinum track and two silver connection wires. For high accuracy temperature measurements, the PRT sensor is usually connected in 4-wire mode to a resistance measuring instrument.

From a gas turbine engine perspective, the miniature thin-film PRT offers two primary advantages over the thermocouple. Firstly, the need for a reference junction and associated hardware is removed, reducing system complexity and eliminating a potential source of measurement error. Secondly, the signal levels are usually far higher compared to a thermocouple and so are less likely to be affected by noise from the test environment. Additionally, the calibration retention is generally expected to be better for the PRT, with lower susceptibility to drift over the useful temperature range. For engine applications, the primary limitation of the thin-film PRT is its temperature capability, which is typically restricted to $500^{\circ} \mathrm{C}$. Consideration should also be given to potential hysteresis in the resistance-temperature characteristic, as well as the possible impact of thermal cycling. For example, Gam et al (2011) have reported the measurement of hysteresis for 30 thin-film PRT sensors when cycled between 0 and $500{ }^{\circ} \mathrm{C}$. A range of hysteresis levels was observed in these devices (between 16 and $156 \mathrm{mK}$ ) although after six repeat thermal cycles these reduced to less than $53 \mathrm{mK}$ for all sensor types tested. In the current work a similar hysteresis study has been conducted (over a smaller temperature range) and is presented in the next section.

\subsubsection{Basic characterization of thin-film PRT behaviour The work reported in} this article considers the temperature measurement performance of PRT-based probe designs. The static calibration repeatability of thin-film PRT sensing elements has been investigated in order to provide confidence in the interpretation of the wind-tunnel results obtained. The temperature range considered in this exercise is thus limited to the 
expected flow temperature range used during the dynamic calibrations. If the sensors were to be used over a wider temperature range (as may be the case in engine test campaigns) this exercise would need to be repeated over an appropriate temperature range (see for example Gam et al, 2011). Additionally, the opportunity was taken to establish whether mechanical vibration leads to a change in static calibration behaviour.

(NB: Appendix A contains a description of the equipment used to perform static calibrations of thermal instrumentation; Table 1 and Table 2 contain summaries of the measurement uncertainties in all aerodynamic tests reported in this article.)

2.1.1.1. Static calibration performance In order to characterize the repeatability of the thin-film PRT devices selected for this work, a set of repeat static calibration tests have been conducted on a single PRT sensor. This investigation has involved repeatedly calibrating a sensor in the range 5 to $25^{\circ} \mathrm{C}$ over a period of approximately four months. Fig. 2 shows the results of this study, with the data for 9 repeat tests being presented as the change in indicated temperature relative to the initial calibration. The data show that the maximum deviation in indicated temperature is less than $0.015^{\circ} \mathrm{C}$. This figure is comparable to the absolute calibration uncertainty of the equipment used.

2.1.1.2. Vibration testing Since the target application for the thin-film PRT sensor is a gas turbine engine, the influence of environmental variables such as vibration on calibration retention is important. The impact of vibration on the static calibration of a miniature PRT has been determined by using an electro-dynamic shaker to generate known levels of vibration. An initial static calibration of the PRT under test was obtained before subjecting it to vibrations of a particular magnitude, frequency and duration. The static calibration of the sensor was then repeated after 3, 6 and 9 hours of vibration. The PRT device was mounted on the shaker face-plate and subjected to a $100 \mathrm{~Hz}$ sinusoidal displacement that yielded a peak acceleration of $20 \mathrm{~g}$. This 
mounted instrumentation. The results of this study are shown in Fig. 3, where data is presented as a change in indicated temperature relative to the initial calibration obtained prior to shaking. The sensor appears to show little evidence of being affected by the vibration, with the difference in repeat calibrations being similar to the variability seen in repeat static calibrations discussed in Section 2.1.1.

\subsection{Stagnation tube designs}

In applications involving high speed gas flows, the temperature indicated by a probe depends not only on the sensing element behaviour but also on the design of the shroud/installation into which the sensor is mounted. For the measurement of flow stagnation temperature, the sensing element is ideally located in an environment where the flow velocity is low enough to reduce temperature recovery effects to low levels (typically less than a Mach number of 0.15). However, in gas turbine engine applications, the provision of such a condition is complicated by the generally high-subsonic Mach number of the flow, and the need to have both compact and mechanically robust probe designs. In order to achieve this, the body of the shroud surrounding the sensor commonly takes on the form of an aspirated metal stagnation tube.

\subsubsection{Aerodynamic design considerations Fig. 4 shows a schematic cross-sectional} diagram of a thermocouple-based stagnation probe head that might currently be utilised in gas turbine engine tests (similar to those considered by Hottel and Kalitinsky, 1945, Moffat, 1961 and Zeisberger, 2007); note that the outer diameter of a such design is typically in the range of 3 to $6 \mathrm{~mm}$. This design features a thermocouple sensing element that is shrouded by a vented stagnation tube. When inserted into a gas flow, the probe is positioned such that the inlet plane of the stagnation tube is approximately normal to the free stream flow direction. As gas enters the probe it undergoes a rapid 
free-stream velocity. At a given oncoming flow condition, the flow speed over the thermocouple junction is primarily determined by the inlet to exit area ratio of the stagnation tube. An equivalent probe-head that uses a thin-film PRT sensor is shown in Fig. 5. This design is referred to as the Baseline PRT probe throughout this article. The sensing element is bonded to a short ceramic tube (mullite) through which the two silver connecting wires pass in fine-bore holes. The ceramic tube is bonded into the stagnation tube and 4-wire extension connections are made in this region. The use of a ceramic mounting offers electrical isolation as well as providing a support of relatively low thermal conductivity which is important for reducing heat conduction effects. The stagnation tubes are typically made of stainless steel, although this depends primarily on the temperature range to which the device will be subjected.

2.2.2. Thermal design considerations In creating a robust and compact temperature instrument, traditional probe designs (such as that shown in Fig. 4) have tended to be affected by thermal conduction between the temperature sensing element and its supporting structure (Moffat, 1961, Zeisberger, 2007). The conduction is driven by a temperature difference between fluid within the stagnation tube and the low recovery temperature of the flow on the probe outer surfaces. The heat transfer situation within a generic probe-head is depicted in Fig. 6. The gas temperature within the stagnation tube is high (near to $T_{o, \infty}$ ), while the recovery temperature of the fluid flowing over the exterior of the probe is low (see Section 4.1). Depending on the balance of heat transfer coefficients over the wetted area of the device, and the thermal resistance of the support structure, heat is transferred away from the sensor causing an under-read in the indicated stagnation temperature. Essentially, the sensing element temperature is determined by a heat balance between convective and conductive heat transfer mechanisms. Because the convective heat transfer is sensitive to the Reynolds number of the flow, the under- 
read in indicated temperature tends to be sensitive to flow conditions. Therefore, in order to achieve high measurement accuracy without aerodynamically calibrating the probe as a function of Reynolds number, the impact of this conduction-based error must be reduced. One common approach to this problem (Zeisberger, 2007, Moffatt, 1961) is to increase the convective heat transfer coefficient between the fluid and the sensing element; this is achieved by increasing the flow velocity in the aspiration tube by enlarging the vent hole area. However, the benefits of this approach are eventually negated by the temperature recovery effects on the sensor itself. Another option that can reduce thermal conduction effects is to construct the probe from materials that have a particularly low thermal conductivity, although in general this is complicated by the requirements of probe robustness and temperature rating.

\section{Experimental approach for characterising probe aerodynamic performance}

Various stagnation temperature probe designs have been tested in a high-pressure air facility that can produce ambient temperature jet flows with a Mach number of up to 0.8. The facility can be configured to produce confined jet flows at either ambient static pressure or at elevated static pressures up to 4 bar absolute. This arrangement enables Reynolds number effects to be investigated. The aerodynamic calibration facility is shown schematically in Fig. 7 and consists of a compressor system that delivers dry air $\left(-40^{\circ} \mathrm{C}\right.$ dew point) at a pressure of up to 14 bar to a $100 \mathrm{~m}^{3}$ receiver. The supply of this air to the working section is controlled by electronically actuated valves and is finally delivered, via a $150 \mathrm{~mm}$ diameter pipe, to a convergent nozzle with a $50 \mathrm{~mm}$ throat diameter. The compressor can deliver up to $1 \mathrm{~kg} / \mathrm{s}$ of air, which enables continuous running over the range of flow conditions investigated. The leading edge of the probe 
throat plane. The open area of the exit plate (shown in Fig. 7) can be manipulated manually between tests in order to allow adjustment of the working section static pressure. In this work, the ratio of nozzle exit area to exit plate flow area $\left(A_{N} / A_{E}\right)$ has been set to values of 0.03 (ambient static pressure condition of 1 bar), $0.64,0.74$ and 0.81. Details of the typical jet-flow uniformity and other aspects of the facility are provided by Wilson et al (2012).

\subsection{Facility instrumentation}

The flow conditions in the jet are established from measurements of stagnation pressure and temperature in the feed pipe, and the static pressure at the nozzle exit plane. The stagnation quantities are measured in a low velocity (Mach number less than 0.15 ) region of the feed pipe where the impact of recovery effects is reduced to a low level. There is assumed to be no change in flow stagnation properties between the working section and the measurement location.

The feed stagnation temperature is measured using a commercially available, passively aspirated thin-film PRT device that is monitored by a Pico PT-104 resistance measurement instrument. This PRT is connected in 4-wire mode with a sense current of $0.5 \mathrm{~mA}$. The probe and the associated data acquisition device are calibrated as a system against a traceable temperature standard. At atmospheric conditions, the measurement uncertainty in flow stagnation temperature is less than $0.1 \mathrm{~K}$ (see Appendix A).

The feed stagnation pressure is determined using a conventional Pitot tube, whilst the static pressure at the nozzle exit plane is obtained via a tapping in the working section side-wall. These measurements allow calculation of the jet Mach number from the isentropic pressure relation. Together with knowledge of the stagnation temperature, the measurements also enable the flow Reynolds number to be established. The static 
connected to a National Instruments voltmeter. The pressure transducers are calibrated over their operational range using a variable pressure source, traceable to a measurement standard. At typical operating conditions, the uncertainty in rig pressure measurements is estimated at 2 mbar. This corresponds to Mach and Reynolds number uncertainties of approximately 0.004 and 400 respectively (see Appendix A).

\subsection{Probe temperature recovery factor definition}

The stagnation temperature measurement performance of various probe arrangements has been quantified by testing in the high-pressure air facility described above. The probe performance is presented as a probe temperature recovery factor, $R_{p}$, defined as follows:

$$
R_{p}=\frac{T_{i}-T_{\infty}}{T_{o, \infty}-T_{\infty}}
$$

In this definition, the free-stream static temperature, $T_{\infty}$, is determined from the measured flow stagnation temperature as follows:

$$
T_{\infty}=\frac{T_{o, \infty}}{1+\frac{\gamma-1}{2} M_{\infty}^{2}}
$$

while the free-stream Mach number, $M_{\infty}$, is determined from the measured flow stagnation pressure, $P_{o, \infty}$ and jet static pressure, $P_{\infty}$, thus:

$$
M_{\infty}=\left[\left[\left(\frac{P_{o, \infty}}{P_{\infty}}\right)^{\frac{\gamma-1}{\gamma}}-1\right] \frac{2}{\gamma-1}\right]^{\frac{1}{2}}
$$

\subsection{Aerodynamic test conditions}

Probes have been tested over a range of flow Mach numbers between 0.3 and 0.75 , and with flow stagnation temperatures in the range 5 to $20^{\circ} \mathrm{C}$ (this being dependent on atmospheric temperature). The range of Mach number conditions considered is typical of an engine gas-path measurement environment, whilst the temperature range 
stage fan. The angular sensitivity of the probes has also been investigated over a range of flow incidence angles from 0 to 30 degrees.

Note that uncertainty estimates for all relevant experimental quantities are summarised in Appendix A.

\subsection{Probe installation arrangements}

Probes have been tested in both sting-mounted and rake-mounted arrangements; these are shown schematically in Fig. 8. The rake-mounted arrangement simulates a typical engine measurement geometry, both in terms of the aerodynamic situation around the probe-head and the thermal boundary conditions to which it is subjected. The stingmounted arrangement essentially allows the probes to be assessed in isolation of any installation effects.

\section{Baseline PRT probe performance}

Fig. 9 shows the measured probe recovery factor, $R_{p}$, for two Baseline PRT devices (shown schematically in Fig. 5) tested in a sting-mounted arrangement at an ambient static pressure condition of $1 \mathrm{bar}\left(A_{N} / A_{E}=0.03\right)$. In one design the probe body is constructed from stainless-steel $(k=16 \mathrm{~W} / \mathrm{mK})$, whilst in the other the probe body is constructed from low thermal conductivity acrylic $(k=0.2 \mathrm{~W} / \mathrm{mK})$. In both cases the probe head has an inlet to exit-vent area ratio of 3.7, and an outer diameter of $5 \mathrm{~mm}$. The device that is constructed from stainless-steel exhibits a probe recovery factor that varies between 0.85 and 0.89 over the Mach number range considered, with a generally increasing trend as Mach number (and also Reynolds number) is increased. In comparison, the data obtained for the acrylic-bodied probe demonstrates a far higher probe recovery factor $(0.96$ to 0.98$)$ as well as less sensitivity to the free-stream condition. 
properties of the stagnation tube, it is apparent that conduction effects have a strong influence on the temperature indicated by this style of probe assembly. The use of a low thermal conductivity material for the probe body is therefore beneficial to the underlying accuracy of the indicated temperature.

Although this result demonstrates the importance of thermal conductivity in achieving acceptable stagnation temperature measurement performance, acrylic (and plastics in general) is clearly not a suitable material for engine test hardware, which requires a sufficient temperature rating and must also be mechanically robust. It does however confirm that obtaining accurate indicated probe temperature measurements, which require low levels of post-test correction, demands effort in terms of limiting thermal conduction effects. This has led to the development of a new probe design which is described in detail in Section 5.

\subsection{Measurements of recovery temperature on shroud outer wall}

Interestingly, the low probe recovery factors measured for the stainless-steel Baseline PRT device (shown in Fig. 9) are similar in magnitude to the recovery factor for a fully developed turbulent boundary layer over a flat plate, suggesting that in terms of $R_{p}$ the stagnation tube is providing little effect. It is clear that this behaviour is connected to thermal conduction within the probe assembly; however, the particularly low value of $R_{p}$ is still surprising. In an effort to understand this phenomenon, an experiment has been conducted to examine the adiabatic wall temperature on the outer surface of a probe-like body, ie. a cylinder with its axis aligned to the oncoming flow direction. This information is important as it is the adiabatic wall temperature (gas recovery temperature) around the probe that determines the thermal boundary condition driving heat conduction within the sensor. 
experiment. The diagram shows a thin-walled tube $(0.2 \mathrm{~mm}$ wall thickness $)$ that has 8 miniature thermocouples attached at various axial locations along its inner surface. The tube represents a near-adiabatic wall condition, with low heat transfer into the stagnant air pocket and low levels of conduction. The measured wall temperatures, expressed in terms of recovery factor, are presented in Fig. 11 for various free-stream Mach number conditions at an ambient static pressure of 1 bar $\left(A_{N} / A_{E}=0.03\right)$. From this data, a significant variation in the wall temperature recovery factor with axial position can be observed. In the forward region of the cylinder (up to 1 diameter downstream of the leading edge) the temperature recovery factor is particularly low, attaining a value of around 0.6. Beyond this, however, the recovery factor steadily increases, reaching a value of approximately 0.82 in the cylinder's most downstream region. Note that the recovery factor for a fully developed turbulent boundary layer over a flat plate is typically 0.88. Based on schlieren visualisation, the low wall temperature measured around the cylinder leading edge appears to be associated with a region of separated flow. Low temperatures within separated flows are thought to be caused by a number of complex factors that include shear work, pressure-related energy separation in the shear layer, thermal conduction and the recirculatory flow patten. This has a potentially important implication for stagnation temperature probe design, as it is the difference between the adiabatic wall temperature and the flow temperature within the probe head that contributes to the under-read in probe indicated temperature.

\section{A new stagnation temperature probe: the dual-skin device}

The Baseline PRT probe shown schematically in Fig. 5 exhibits a low probe recovery factor, which is driven by the low temperature on the outer wall of the device. Obtaining sufficiently accurate stagnation temperature measurements with this particular probe 
calibration data. Consequently, a new probe arrangement has been devised that is primarily intended to reduce the impact of thermal conduction on the indicated stagnation temperature measurement, but without the need to resort to low thermal conductivity materials. This device allows the benefits of PRT sensors to be realised in a stainless-steel probe-head that is both compact and robust for gas turbine engine applications. This section describes the new design, which is based on a passively aspirated arrangement, and which is referred to as a dual-skin probe. Following the guidance provided by Moffat (1961), the approach has been to produce a probe that has an appropriate accuracy for the particular application, and that consequently requires low levels of aerodynamic calibration and post-test correction.

\subsection{Dual-skin shroud design}

The design of the dual-skin probe has sought to minimise the effects of thermally conducting paths between the sensing element, its support structure and the relatively cold probe outer body. This has been achieved by using an inner cavity within the probe that is continuously purged by stagnated air that enters through the sensing port. A schematic diagram of the dual-skin arrangement is shown in Fig. 12. The miniature PRT sensing element is bonded to a ceramic tube through which the two silver connecting wires are guided. The wires bridge an air-filled gap to the mounting support where fourwire extension connections are made. The key aspect of the dual-skin design is that the PRT sensing element is mounted onto an inner stainless-steel tube that connects onto the outer probe body at the entrance to the sensing chamber only. Near-stagnated air passes over the sensor and through bleed holes in this inner-skin that allow the air to enter the surrounding annular chamber. The aspirating flow is allowed to leave the annular chamber through a further set of vent holes in the outer shroud skin (as in the 
and its mounting assembly are surrounded by high temperature stagnated air, with minimal thermal contact onto the cold outer body of the probe. In seeking to minimise the thermal conduction mechanisms (and thereby the heat flow through the sensor and ceramic support) it is anticipated that the temperature measurement accuracy of this design will show low sensitivity to Reynolds number, since the significance of convective heat transfer to the value of $R_{p}$ is reduced. The inlet to exit-vent area ratios of the prototype dual-skin probe are 1.3 and 2.6, these being for the inner and outer shroud skins respectively.

\subsection{PRT sensing element}

The PRT sensing elements selected for this study are miniature thin-film platinum resistance devices manufactured by Minco Ltd; the sensor dimensions are $1.3 \times 1.0 \mathrm{~mm}$ in plan form, and are approximately $0.5 \mathrm{~mm}$ thick. This device has two silver wires that connect to the platinum element and which are connected to extension wiring in 4-wire configuration, as shown in Fig. 12. The nominal resistance of the sensor is 100 Ohms at $0{ }^{\circ} \mathrm{C}$. The temperature capability of these devices is quoted by the manufacturer to be up to $400^{\circ} \mathrm{C}$. The resistance of the sensor is recorded using a Pico PT-104 resistance data logger connected in 4-wire mode (sense current $0.5 \mathrm{~mA}$ ).

\section{Dual-skin probe stagnation temperature measurement performance}

\subsection{Sting-mounted arrangement}

The performance of the dual-skin probe has initially been investigated in a stingmounted arrangement, as shown in Fig. 8. Although this type of installation is unrepresentative of engine geometry, it usefully enables probe behaviour to be assessed in isolation of installation effects. 
6.1.1. Probe recovery factor as a function of free-stream Mach number and flow incidence angle The measured probe recovery factor of the dual-skin probe is shown in Fig. 13 as a function of free-stream Mach number and incidence angle, these data being for an ambient static pressure condition of 1 bar $\left(A_{N} / A_{E}=0.03\right)$. In general, the recovery performance of the dual-skin device shows little sensitivity to Mach number, with essentially constant $R_{p}$ over the Mach number range considered. At zero flow incidence angle the probe recovery factor consistently lies between values of 0.95 and 0.97 . This level of performance is maintained up to an incidence angle of approximately $20^{\circ}$, with a small decrease in recovery factor to 0.94 evident at $30^{\circ}$ incidence. The dual-skin probe demonstrates several important facets. Firstly, a high probe recovery factor that is comparable to the acrylic bodied Baseline PRT probe data shown in Fig. 9. Secondly, a near constant probe recovery factor over a range of Mach numbers typical of gas turbine engine applications. Thirdly, a low sensitivity to flow incidence angle. The high probe recovery factor and its relative insensitivity to free-stream conditions are particularly important, as this suggests that a low level of post-test measurement correction is required, and that any correction is relatively insensitive to operating condition. Consequently, it is feasible that systematic aerodynamic calibration is unnecessary with this design, with consequent relief on cost and time resources.

6.1.2. Probe recovery factor as a function of Reynolds number The recovery factor of the dual-skin probe has also been measured over a range of Mach numbers at different static pressure conditions, allowing the impact of Reynolds number to be assessed. The results of this investigation are shown in Fig. 14 for the case of zero flow incidence angle. The data is presented for 4 different exit plate area ratios (defined in Section 3) and plotted against Reynolds number based on the probe outer diameter. For the range of flow conditions tested, it is clear that the dual-skin device displays a low sensitivity to 
Reynolds number, with the probe recovery factor varying between values of 0.95 and 0.97 for Reynolds numbers of 30,000 and 150,000 respectively. This insensitivity had earlier been forecast based on the consideration of probe thermal boundary conditions, and in particular the reduced significance of convective heat transfer effects (see Section $5.1)$.

The Reynolds insensitivity exhibited by the dual-skin device is in contrast to the performance of the Baseline PRT probe, which has been shown to be strongly affected by Reynolds number effects. This is illustrated by the data in Fig. 15, which shows recovery factors for both the Baseline and dual-skin PRT probes at ambient $\left(A_{N} / A_{E}\right.$ $0.03)$ and elevated $\left(A_{N} / A_{E} 0.64\right)$ static pressure conditions. In the case of the Baseline device a significant improvement in indicated temperature accuracy is observed at fixed Mach number for an increasing flow Reynolds number condition. For example, at a Mach number of 0.75 , an increase in $R_{p}$ from 0.88 to 0.91 is seen over a Reynolds number range of 100,000 to 200,000 . This improvement is attributed to an increase in the convective heat transfer coefficient at the sensor surface, which acts to compensate for heat losses from the sensor due to conduction effects.

\subsection{Rake-mounted arrangement}

In gas turbine engine testing the use of instrumented rakes is usually employed, these typically being radial spokes to which several (perhaps as many as 15) individual sensors are attached (see, for example, Zeisberger 2007). The rake body often has an aerodynamic profile in order to reduce the impact of the intrusive rake on the machine performance. The positioning of instrumentation on a rake can have both an aerodynamic and a thermal impact on the measurement performance. Consequently, the behaviour of a dual-skin probe has been investigated when it is mounted in a rake- 
6.2.1. Probe recovery factor as a function of free-stream Mach number and probe protrusion length Fig. 16 shows the impact of probe protrusion length, $L$, on the recovery performance of a rake-mounted dual skin probe tested over a range of Mach numbers at an ambient static pressure of 1 bar $\left(A_{N} / A_{E} 0.03\right)$. For the various protrusions tested, the data show that the measured probe recovery factor, $R_{p}$, lies between values of 0.95 and 0.97 . These values are consistent with recovery factors measured for the sting-mounted dual-skin probe, which are plotted in Fig. 13. Based on this data, the performance of the dual-skin device is considered to be independent of installation arrangement.

The independent performance of the dual-skin probe with installation arrangement is in stark contrast to the behaviour of the Baseline PRT probe, which has been shown to be sensitive to mounting configuration. This is illustrated by the data in Fig. 17, which shows recovery factors for both sting and rake mounted ( $L=3 \mathrm{~mm}$ ) probe arrangements. The data presented essentially indicate the relative sensitivity of the two probe designs to thermal conduction effects. For the Baseline probe, the increased thermal resistance offered by the rake mounting impedes conduction losses and yields a notable improvement in indicated temperature accuracy. In fact, at a Mach number condition of 0.75 , an increase in $R_{p}$ from 0.88 to 0.93 is seen between the sting and rakemounted arrangements. For the dual-skin probe, however, the inherent insensitivity of the design to thermal conduction means that no change in indicated temperature is observed between the sting and rake-mounted installations.

\section{Conclusions}

This article considers the issue of stagnation temperature measurement in the gas path of gas turbine engines, and in particular the desire to improve fundamental measurement 
to the test environment, the performance of particular temperature sensors and the means of supporting the sensing element in a compact and robust probe device. Engine test experience related to the use of thermocouples in this area has led to the desire to apply platinum resistance thermometers to particular regions of the gas path. Initial tests of a simply constructed PRT-based stagnation temperature probe have revealed that thermal conduction plays an important role in establishing how close the indicated probe temperature is to the actual fluid stagnation temperature. In response to this, a new probe design has been developed that is intended to reduce the impact of thermal conduction between the sensor and probe body (relative to the simple probehead design). The new probe design uses a double shroud assembly that allows the temperature sensor to be thermally isolated from the relatively cold outer parts of the probe-head. This new design exhibits several desirable performance characteristics including a high probe recovery factor (typically 0.97 at ambient pressure conditions) and low angular sensitivity (up to 20 degrees flow incidence). The probe recovery factor measurements also show low sensitivity to Mach and Reynolds numbers. Additionally, the performance in both sting and rake mounted assemblies is similar, suggesting that the measurement performance of the probe is largely independent of installation geometry.

\section{Nomenclature}

$A_{E} \quad$ working section exit choke open area

$A_{N} \quad$ nozzle throat area

$k \quad$ thermal conductivity

$L \quad$ probe protrusion length

$M_{\infty} \quad$ free-stream Mach number 
$P_{o, \infty} \quad$ free-stream stagnation pressure

$P R \quad$ component pressure ratio

$R_{p} \quad$ probe temperature recovery factor

$T_{i} \quad$ measured (indicated) temperature from probe under test

$T_{\infty} \quad$ free-stream static temperature

$T_{o, \infty} \quad$ free-stream stagnation temperature

$T_{o, \text { inlet }}$ component inlet stagnation temperature

$T_{o, e x i t} \quad$ component exit stagnation temperature

$\gamma \quad$ specific heat ratio

$\eta \quad$ component isentropic efficiency

\section{Appendix A: Measurement Uncertainties}

Based on the approach described by Coleman and Steel (1995), experimental uncertainties for all measurements reported in this article have been estimated. These consist of bias and precision values that are reported at the $95 \%$ confidence limit. All temperature instrumentation used and tested in this work has been calibrated against UKAS traceable reference equipment at Loughborough University. The temperature calibration equipment consists of a semi-standard platinum resistance probe, a stirred liquid temperature controlled bath (Isotech Europa) and a resistance meter (Isotech TTI7). Pressure instrumentation has been calibrated using a G.E. Measurement \& Control PACE6000 device, which provides an accurate and controllable pressure source that is traceable to a measurement standard.

Measurement biases have been estimated from the residual uncertainty associated with the calibration standard and the installed uncertainty relating to the rig 
repeat calibration procedures (typically 10 repeats) and then applying the Student tdistribution to establish the standard deviation. Table 1 summarises the uncertainties in the primary measurements associated with the aerodynamic calibration of stagnation temperature probes. Table 2 summarises the uncertainties in derived experimental values such as flow Mach number and probe recovery factor.

Table 1. Uncertainties in measured variables calculated for a Mach 0.6 test condition.

\begin{tabular}{l|c|c|c} 
variable & bias uncertainty & precision uncertainty & expanded uncertainty \\
\hline probe indicated temperature $(\mathrm{K})$ & 0.02 & 0.008 & 0.02 \\
flow stagnation temperature $(\mathrm{K})$ & 0.07 & 0.01 & 0.08 \\
flow stagnation pressure (mbar) & 0.2 & 2.0 & 2.0 \\
static pressure (mbar) & 0.2 & 2.0 & 2.0
\end{tabular}

Table 2. Uncertainties in derived experimental results calculated for a Mach 0.6 test

\begin{tabular}{|c|c|c|c|}
\hline variable & bias uncertainty & precision uncertainty & expanded uncertainty \\
\hline jet Mach number & 0.0004 & 0.004 & 0.004 \\
\hline recovery factor & 0.004 & 0.0007 & 0.004 \\
\hline Reynolds number & 50 & 400 & 400 \\
\hline
\end{tabular}

\section{References}

Coleman H and Steele W 1995 Engineering application of experimental uncertainty analysis AIAA Journal 33 (10) p.p.1888-96

Franz A 1940 Pressure and temperature measurements in supercharger investigations $N A C A-T M-953$

Gam K S, Yang I and Kim Y-G 2011 Thermal hysteresis in thin-film platinum 
Glawe G, Simmons F and Stickney T 1956 Radiation and recovery corrections and time time constants of several chromel-alumel thermocouple probes in high-temperature, high-velocity gas streams $N A C A-T N-3766$

Hottel H and Kalitinsky A 1945 Temperature Measurements in High-velocity Air Streams J. of Applied Mechanics A25-A32

Moffatt R 1961 Gas Temperature Measurement (General Motors Research Laboratories, Michigan)

Willbanks C 1973 Recovery characteristics of a single-shielded self-aspirating thermocouple probe at low pressure levels and subsonic speeds J. of Physics E: Scientific Instruments 6 (11)

Wilson A, Ireland P, Stevenson R, Thorpe S and Martin D 2012 A robust radial traverse temperature probe for application to a gas turbine HP/IP stage ASME paper GT2012-68331, ASME Turbo Expo (Copenhagen)

Zeisberger A 2007 Total Temperature Probes for Turbine and Combustor Applications International Society for Air Breathing Engines ISABE-2007-1108 
Figure 1. A graph showing the impact of a $1 \mathrm{~K}$ error in stage temperature rise on the measured isentropic efficiency of a compressor (inlet stagnation temperature of $300 \mathrm{~K}$ ).

Figure 2. Repeat calibration tests of a miniature thin-film PRT sensor plotted relative to an initial reference calibration (data acquired over a period of 4 months).

Figure 3. Repeat calibration tests of a miniature thin-film PRT sensor, after periods of 3,6 and 9 hours of vibration at $100 \mathrm{~Hz}$, plotted relative to an initial reference calibration.

Figure 4. A schematic diagram of a typical self-aspirating stagnation thermocouple probe.

Figure 5. A schematic diagram of the Baseline PRT probe (note that stagnation tubes constructed of both stainless steel and acrylic have been tested in this work).

Figure 6. A schematic diagram of heat flows in a generic stagnation temperature probe (red arrows indicate heat transfer paths).

Figure 7. A schematic diagram of the aerodynamic calibration facility for characterising probe recovery factors.

Figure 8. Schematic diagrams of rake-mounted (left) and sting-mounted (right) probe arrangements (also showing the definition of the probe protrusion length, $L$, for the rake-mounted case).

Figure 9. A graph showing the measured probe recovery factor, $R_{p}$, of the Baseline PRT probe design as a function of free-stream Mach number for both stainless-steel and 
Figure 10. A schematic diagram of the thin-walled stainless steel cylinder used for determining the recovery factor on the outer surfaces of stagnation tube models.

Figure 11. Measurements of the temperature recovery factor on a cylindrical body as a function of axial position (oncoming flow aligned to the cylinder axis, ambient static pressure).

Figure 12. A schematic diagram of the dual-skin PRT probe-head in sting-mounted configuration (note that the probe overall diameter is typically $5 \mathrm{~mm}$ ).

Figure 13. Probe recovery factor, $R_{p}$, of the dual-skin probe as a function of flow incidence angle and free-stream Mach number (sting-mounted, ambient static pressure).

Figure 14. Probe recovery factor of the dual-skin probe as a function of flow Reynolds number (sting-mounted, zero flow incidence, various static pressure conditions).

Figure 15. Probe recovery factor, $R_{p}$, of the dual-skin probe compared to the Baseline PRT probe at ambient $\left(A_{N} / A_{E} 0.03\right)$ and elevated $\left(A_{N} / A_{E} 0.64\right)$ static pressure conditions (sting-mounted, zero flow incidence).

Figure 16. Probe recovery factor, $R_{p}$, of the dual-skin probe as a function of protrusion from the rake body (rake-mounted, zero flow incidence, ambient pressure).

Figure 17. Probe recovery factor, $R_{p}$, of the dual-skin probe compared to the Baseline PRT probe for both sting and rake mounted configurations (zero flow incidence, 